\title{
Atlas of Human Malaria
}

Giovanni Swierczynski and Maria Gobbo (Authors), published by Az Color s.r.l., Sirmione, Italy (Printed in April 2007), 203 p, ISBN 978-88-902892-0-0.

The Atlas of Human Malaria (book size: 14 by 9 inches) is an illustrated atlas of malaria parasites infecting humans around the world and is written in double language, English and Italian. The book contains a total of 452 highest quality color photomicrographs ( 6 by 4 inches), each with precise description and explanations, and 11 figure or illustration plates (full pages). The Atlas covers mainly the 4 species of human-infecting Plasmodium species, i.e., Plasmodium falciparum, $P$. vivax, $P$. malariae, and $P$. ovale. Every developmental stage of the parasite is very well represented, from the classical morphologic forms to the parasite stages that are difficult to interpret and may pose diagnostic problems. The book also briefly presents accidental parasites or bacteria that can occur in the human blood, such as Babesia microti and other Babesia species, microfilariae, and Borrelia species. In addition to the photomicrographs, the book contains concise texts describing highly useful information on the life cycle, biology, epidemiology, and diagnosis of human malaria. With regard to the diagnosis, the authors have provided an excellent review of the techniques for preparing well stained blood films.

The Atlas is unique in that it provides various types of artifacts that can be accidentally present in human blood films and can be confused with malaria or other blood parasites. The book also contains 4 tables presenting detailed information on the geographical distribution of human malaria, comparative notes on thin and thick blood films, and morphological characteristics of different Plasmodium species. At the end of the book, 18 textbooks or manuals and 83 selected references for further reading are provided.

Malaria continues to be one of the 6 major tropical diseases designated by the World Health Organization; malaria, schistosomiasis, leishmaniasis, trypanosomiasis, filariasis, and leprosy. Malaria is an important health problem in over 40 countries with 2.4 billion people that comprises $40 \%$ of the world population. Each year 500 million new malaria cases occur and 1.52.7 million cases die annually. Therefore, the issue of laboratory diagnosis of malaria is highly important and provision of good guidelines in the laboratory procedures and morphological differentiation of parasites is definite and essential.

In this respect, the Atlas of Human Malaria provides a new level of assistance in the laboratory diagnosis of human malaria. The color photomicrographs, explanations, and text descriptions are all highest quality and most updated ones. The book will be highly useful for young malariologists working in the field or laboratory, tropical medicine doctors, laboratory technologists, and students.

Jong-Yil Chai

Department of Parasitology and Tropical Medicine, Seoul National University College of Medicine, Seoul 110-799, Korea (cjy@snu.ac.kr) 